\title{
Differential effects of dietary diversity and maternal characteristics on linear growth of children aged 6-59 months in sub-Saharan Africa: a multi-country analysis
}

\author{
Dickson A Amugsi ${ }^{*}$, Zacharie T Dimbuene, Elizabeth W Kimani-Murage, Blessing Mberu \\ and Alex C Ezeh \\ African Population and Health Research Center, APHRC Campus, PO Box 10787-00100, Nairobi, Kenya
}

Submitted 7 August 2016: Final revision received 14 November 2016: Accepted 21 November 2016: First published online 9 January 2017

\begin{abstract}
Objective: To investigate the differential effects of dietary diversity (DD) and maternal characteristics on child linear growth at different points of the conditional distribution of height-for-age $Z$-score (HAZ) in sub-Saharan Africa.

Design: Secondary analysis of data from nationally representative cross-sectional samples of singleton children aged 0-59 months, born to mothers aged 15-49 years. The outcome variable was child HAZ. Quantile regression was used to perform the multivariate analysis.

Setting: The most recent Demographic and Health Surveys from Ghana, Nigeria, Kenya, Mozambique and Democratic Republic of Congo (DRC).

Subjects: The present analysis was restricted to children aged 6-59 months (n 31604 ).

Results: DD was associated positively with HAZ in the first four quantiles (5th, 10th, 25th and 50th) and the highest quantile (90th) in Nigeria. The largest effect occurred at the very bottom ( 5 th quantile) and the very top (90th quantile) of the conditional HAZ distribution. In DRC, DD was significantly and positively associated with HAZ in the two lower quantiles (5th, 10th). The largest effects of maternal education occurred at the lower end of the conditional HAZ distribution in Ghana, Nigeria and DRC. Maternal BMI and height also had positive effects on HAZ at different points of the conditional distribution of HAZ.

Conclusions: Our analysis shows that the association between DD and maternal factors and HAZ differs along the conditional HAZ distribution. Intervention measures need to take into account the heterogeneous effect of the determinants of child nutritional status along the different percentiles of the HAZ distribution.
\end{abstract}

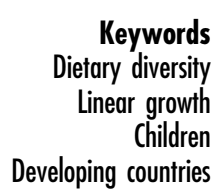

Child undernutrition is a major public health problem, particularly in developing countries, and efforts at reducing it are central to economic and social development in any country because of the dire ramifications of undernutrition on the physical, psychological and mental development of individuals over the life course ${ }^{(1-3)}$. There is also a link between undernutrition and higher morbidity and mortality incidence in children. For example, a severely stunted child faces four times higher risk of dying and a severely wasted child is at nine times higher risk $^{(3-5)}$. Overall, poor child nutritional status is estimated to contribute to more than one-third of all child deaths, although it is rarely listed as the direct cause ${ }^{(1,3)}$. Besides, there is a lethal interaction between undernutrition and common childhood infections ${ }^{(1,3)}$. Thus, undernutrition puts children at elevated risks of severe illnesses due to common childhood infections such as pneumonia, diarrhoea, malaria, HIV/AIDS and measles ${ }^{(1,3)}$. The preceding discussion suggests the imperativeness of having broad knowledge and understanding of the correlates of child malnutrition, to inform appropriate interventions to arrest the problem. The subsequent paragraphs discuss in detail the various correlates of child malnutrition.

Dietary diversity (DD), defined as the sum of food groups consumed over a period of $24 \mathrm{~h}$, has been documented as a valid and reliable indicator of dietary adequacy for young children ${ }^{(6-8)}$. Therefore, DD is a reasonably easy-to-measure proxy variable for young children's nutrient intake ${ }^{(9-11)}$ and the WHO uses DD as one of the key indicators to assess child feeding practices ${ }^{(12)}$. A number of studies have demonstrated the relationship between DD and child growth $^{(13,14)}$. 
In examining data from eleven countries in Africa and Latin America, Arimond and Ruel ${ }^{(14)}$ observed that DD has a significant positive effect on height-for-age $Z$-score (HAZ). DD was also found to interact significantly with other sociodemographic factors. The authors concluded that there is an association between child DD and nutritional status that is independent of socio-economic factors, and that DD may indeed reflect diet quality ${ }^{(14)}$. DD has also been established as a significant predictor of child growth in Ghana, as illustrated by an analysis of Ghana Demographic and Health Survey (DHS) data from children aged 6-36 months ${ }^{(13)}$. However, the limitation of the above studies is that they mainly examined the average effect of DD on child growth. This may not represent a comprehensive picture of the effect of DD on child growth. This is the gap the present study intends to fill.

Maternal BMI and height have been identified several times in the literature as a set of critical factors affecting child growth $^{(2,15-19)}$. A study in Ethiopia showed that both maternal BMI and height were associated positively with children's nutritional status ${ }^{(19)}$. Similar positive associations were observed in Brazil and the positive association between maternal height and child HAZ was in a linear fashion $^{(15)}$ : mothers who were tall tended to have taller children compared with mothers who were short. However, another study using quantile regression (QR) methods observed that maternal BMI influenced child stunting in non-linear ways ${ }^{(17)}$. This is not surprising because the two studies used different analytical methodologies. The positive association between maternal BMI and child growth was also observed in Ghana ${ }^{(20)}$. A related study in India indicated differential effects of maternal nutrition at different points of the conditional distribution of the child anthropometric $Z$-scores ${ }^{(2)}$. To demonstrate the importance of maternal height to child growth, Ozaltin et al. ${ }^{(21)}$ analysed 109 DHS from fifty-four African countries and found that a $1 \mathrm{~cm}$ increase in maternal height was associated with a decrease in the risk of child stunting and consequential effects such as mortality.

Similarly, maternal age and parity are important factors that affect child growth. Fenske et $a l .{ }^{(17)}$ observed that maternal age has a significant effect on childhood stunting. Two other studies established the relationship between maternal age and child nutritional status ${ }^{(22,23)}$. For example, in India, undernourishment was greater in children whose mothers were aged 26-30 years than in the other extremes of reproductive ages ${ }^{(22)}$. Relatedly, mothers in their early 20 s tended to have children with poorer health outcomes, including nutritional status, while children of older mothers tended to suffer less from stunting ${ }^{(23,24)}$. Although there is paucity of literature on the effects of maternal parity on child nutritional status, one study in urban poor settlements in Kenya observed a strong association between maternal parity and child growth ${ }^{(25)}$.

Another important factor of child nutritional status is maternal education. There is substantial evidence that children whose mothers are educated tend to have better nutritional status compared with children of mothers with no education. Two studies using QR have illustrated that an improvement in maternal education was associated with a dramatic increase in child $\mathrm{HAZ}^{(2,17)}$. A study in Sri Lanka revealed that parental education, in the midst of other sociodemographic factors, had a larger effect on child height at the upper quantiles than at the lower quantiles $^{(26)}$. Furthermore, studies that examined the mean effect of maternal education have also elucidated the strong positive association between maternal education and child growth ${ }^{(16,27,28)}$. In Indonesia, mothers who never attended formal education had children who were stunted $^{(16)}$, while in Ghana it was observed that increase in mother's years of education was associated with decreased malnutrition ${ }^{(20)}$. Related to maternal education is maternal occupation. Even though a woman's employment enhances the household's accessibility to income, thereby making nutritious food available to children and consequently improving child growth ${ }^{(19,27,29)}$, it may also have negative effects on the nutritional status of children as it reduces a mother's time for childcare ${ }^{(28)}$. Some studies have revealed that the most malnourished children have mothers who work outside their home ${ }^{(30,31)}$. Nevertheless, the general observation is that maternal employment has a positive effect on child nutritional status ${ }^{(32,33)}$, although mechanisms through which this effect operates remain unclear.

The literature reviewed above focuses almost exclusively on mean effects of DD and maternal characteristics on child nutritional status. Statistical analyses that simultaneously examine the effects of child DD and maternal characteristics at different points of the distribution of child growth score are, to the best of our knowledge, still missing. As sociodemographic background variables and policy interventions may affect child nutrition differently at different points of the conditional nutritional distribution, it is significant to conduct analyses that will give a comprehensive and complete picture of these effects. Using QR, the present study explored the effects of DD and maternal characteristics on child linear growth at different points of the conditional distribution of HAZ.

\section{Methodology}

\section{Design and data sources}

The study used the most recent DHS ${ }^{(34)}$ data from Ghana (2014), Kenya (2014), Nigeria (2013), Mozambique (2011) and Democratic Republic of Congo (DRC; 2013). The design of the DHS is identical across all participating countries, making possible comparisons between and across countries. The DHS utilizes a two-stage sample design $^{(35-39)}$. The first stage involved the selection of sample points or clusters from an updated master sampling frame constructed from the national Population 
and Housing Census of the respective countries. The clusters were selected using systematic sampling with probability proportional to size. Household listing was then conducted in all the selected clusters to provide a sampling frame for the second-stage selection of households. The second stage of selection involved the systematic sampling of the households listed in each cluster, and households to be included in the survey were randomly selected from the list. The rationale for the second-stage selection was to ensure adequate numbers of completed individual interviews to provide estimates for key indicators with an acceptable precision. All men and women aged 15-59 and 15-49 years, respectively, in the selected households (men in half of the households) were eligible to participate in the surveys if they were either usual residents of the household or visitors present in the household on the night before the survey.

\section{Study participants}

The study population comprised nationally representative cross-sectional samples of singleton children aged 0-59 months, born to mothers aged 15-49 years, from five countries in sub-Saharan Africa. Information on children was obtained through a face-to-face interview with mothers. Height was measured with an adjustable measuring board calibrated in millimetres. Children younger than 24 months were measured lying down (recumbent length) on the board, while standing height was measured for older children. Weight was measured using calibrated electronic scales. The measurements were converted into $Z$-scores based on the 2006 WHO growth standards ${ }^{(40)}$. Due to the inclusion of child DD as a key variable, the present analysis was restricted to children aged 6-59 months. This age group was used because, according to the WHO recommendation ${ }^{(41)}$, all children are expected to be exclusively breast-fed until 6 months of age. Hence, the DD indicator may not apply to them. The choice is also in line with the recommended age limit for the construction of the DD indicator for children ${ }^{(42)}$. The total samples used in the current analysis were: Ghana, $n$ 1388; Nigeria, $n$ 13823; Kenya, $n$ 4623; Mozambique, $n$ 7025; and DRC, $n 4745$.

\section{Ethics statement}

Ethical approvals were obtained from the national ethics committees of the respective countries before the surveys were conducted. Written informed consent was obtained from every participant before she/he was allowed to take part in the survey. Consent was obtained from parents before their children's measurements were taken. The DHS Program, USA, granted the authors permission to use the data. The data were completely anonymous; therefore the authors did not seek further ethical clearance.

\section{Outcome and explanatory variables}

Outcome measure

The outcome measure was HAZ and was used as an indicator of child linear growth. Children who have heightfor-age below -2 SD from the median height-for-age of the WHO reference population $(\mathrm{HAZ}<-2)^{(40)}$ are considered stunted (chronically malnourished). However, for the purpose of the present study and due to the methodological choice to use QR, HAZ was used in the analysis as a continuous variable.

\section{Explanatory measures}

The explanatory variables were classified into DD score and maternal characteristics. The DD score was created using data from the $24 \mathrm{~h}$ recall of food groups available in the DHS data sets of the respective countries. The general approach was to develop a score that included a point for each of the nutritionally important types of food the child had eaten in the last $24 \mathrm{~h}$. The DD score was constructed by a simple count of food groups consumed by the child over the past $24 \mathrm{~h}$ preceding the interview of the mother, who reported the child's food consumption. Based on the recommended procedure on how the child DD indicator should be constructed ${ }^{(42,43)}$, the food types in the data were regrouped into seven ${ }^{(7)}$ main categories: (i) grains, roots and tubers; (ii) legumes and nuts; (iii) dairy products; (iv) flesh foods and organ meats; (v) vitamin A-rich fruits and vegetables; (vi) eggs; and (vii) other fruits and vegetables. The mothers reported whether or not their children had consumed any of the abovementioned food groups within the last $24 \mathrm{~h}$. A value of 1 was given for the child's consumption of any of the food groups within $24 \mathrm{~h}$, while 0 was assigned for non-consumption. These scores were then summed up to obtain the DD score, ranging from 0 to 7 , which was used in the analysis as a continuous predictor variable. The maternal-level characteristics included BMI (derived by dividing weight in kilograms by the square of height in metres), height, age, parity, education and work status. The following factors were controlled for in the analysis: child-level factors (age and sex) and household-level factors (household wealth and number of household members). The selection of the explanatory variables was based on an extensive review of the literature.

\section{Data analysis}

Estimation of the quantile regression model

The QR method was introduced by Koenker and Bassett $^{(44)}$ as a 'location model' to extend ordinary least squares (OLS), which summarizes the distribution at its grand mean, into a more general class of linear models in which the conditional quantiles have linear form to fully account for the overall distribution of the response variable. To formalize the QR method, consider a real-valued 
random variable $Y$ characterized by the following distribution function ${ }^{(44,45)}$ :

$$
F(y)=\operatorname{Pr}(Y \leq y) .
$$

Then, for any $\tau \in(0,1)$, the $\tau$ th quantile of $Y$ is defined as:

$$
Q(\tau)=\inf \{y: F(y) \geq \tau\} .
$$

The common quantiles $\tau$ from equation (1) are $\tau=0.25$, $\tau=0.50$ and $\tau=0.75$ for the first quartile, the median and the third quartile. Therefore, unlike OLS which minimizes the squared differences around the mean, QR minimizes the weighted absolute difference between the observed value of $y$ and the $\tau$ th quintile of $Y$. It can easily be demonstrated that OLS is nested in $\mathrm{QR}^{(44,45)}$.

\section{Statistical analysis approach}

The present study used the QR method ${ }^{(44)}$ to estimate the effects of DD and maternal characteristics on child linear growth (HAZ), controlling for child-level factors (age, sex) and household-level characteristics (household size, wealth index). Analysis included descriptive and multivariate techniques using the statistical software package Stata version 13. The descriptive analysis explored the characteristics of the sample. This was followed by multivariate analysis to establish the relationship between the predictor variables and the outcome variable. We employed QR to conduct the multivariate analysis to capture the full distribution of the outcome variable. We estimated OLS regressions and QR at the 5th, 10th, 25th, 50th, 75th and 90th quantiles. QR was chosen over OLS regression because we wanted to examine the effects of the predictor variables at different points of the conditional distribution of the outcome variable (HAZ). This is not possible with OLS, as standard OLS regression techniques summarize the average relationship between a set of regressors and the outcome variable based on the conditional mean function $E(y \mid x)$. This provides only a partial view of the relationship, as we might be interested in describing the relationship at different points in the conditional distribution of $y$. Thus, unlike OLS regression, QR provides a complete view of the effect of an independent variable on the outcome variable; therefore, it is possible to identify the more vulnerable groups and devise more effective interventions. In addition, while OLS can be inefficient if the errors are highly non-normal, $Q R$ is more robust to non-normal errors and outliers ${ }^{(44)}$. QR also provides a richer characterization of the data, thereby illuminating the impact of a covariate on the entire distribution of $y$, not merely its conditional mean.

\section{Results}

\section{Descriptive analysis}

Table 1 presents the characteristics of the samples used in the analysis. Among the five countries, Ghana had children with the highest HAZ (-1.01 (SD 1.30)), while DRC had children with the lowest HAZ (-1.71 (SD 1.81)). The mean DD for each of the countries was as follows: Ghana, 2.16 (SD 1.65); Nigeria, 1.92 (SD 1.74); Kenya, 2.51 (SD 1.79); Mozambique, 2.61 (SD 1.97); and DRC, 1.95 (SD 1.49). The DRC sample contained older children on average (mean age 28.15 (SD 16.25) months) than the four remaining countries. The mothers in the Kenyan sample had the highest number of years of formal education (mean 6.02 (SD 4.38) years), with Mozambique having the lowest (mean 3.26 (sD 3.36) years). The majority of the mothers in Ghana (77\%), DRC (77\%) and Nigeria (69\%) indicated they were working, while a little over half (53\%) in Kenya and less than half in Mozambique (37\%) indicated they were working. Mothers in Ghana tended to have higher mean BMI (23.90 (SD 4.61) $\mathrm{kg} / \mathrm{m}^{2}$ ) than mothers in the remaining four countries. The mean height was almost the same across all the five countries.

\section{Multivariate quantile regression analysis}

Tables 2-8 present the results of the multivariate QR analysis for Ghana, Nigeria, Kenya, Mozambique and DRC, respectively. For the purposes of comparison, OLS estimates are also reported in all of the tables. The OLS estimates indicated a strong effect of child DD in Nigeria and DRC, with HAZ increasing with DD. Maternal education had a strong effect, with HAZ increasing with years of education in four of the five countries, while declining with years of education in one country (Kenya). Maternal age had a positive effect on child HAZ across all countries; while maternal parity had a negative effect on HAZ, with HAZ declining with the increase in maternal parity in all five countries. Maternal work status had a positive effect on HAZ in Nigeria and a negative effect in Kenya. Maternal BMI and height had positive effects on child HAZ in all five countries.

The QR results suggested important differences at different points in the conditional distribution of HAZ. The effect of child DD on HAZ was significant in the first four quantiles (5th, 10th, 25th and 50th) and the highest quantile (90th) in Nigeria. The largest effect occurred at the very bottom ( 5 th quantile) and the very top (90th quantile) of the HAZ distribution. A 1-unit increase in DD was associated with an increase in child HAZ of 0.052 and 0.065 at the 5 th and 90th quantile, respectively. There was no significant effect at the 75th quantile. Stratifying further by child age showed that among those aged 24-59 months, the effect was across all quantiles, with the greatest effect being at the two lower quantiles (5th and 10th) and the highest quantile (90th). Thus, a 1-unit change in DD was associated with a 0·120, 0.124 and $0 \cdot 105$ increase HAZ in the 5th, 10th and 90th quantile, respectively, for children in Nigeria. Conversely, a negative effect of DD on HAZ was observed among children aged 6-23 months at the 50th and 75th quantiles. In DRC, DD was significantly and positively associated with child HAZ 
Table 1 Descriptive analysis of the samples' characteristics

\begin{tabular}{|c|c|c|c|c|c|c|c|c|c|c|}
\hline \multirow[b]{2}{*}{ Variable } & \multicolumn{2}{|c|}{$\begin{array}{c}\text { Ghana } \\
(n \text { 1388) }\end{array}$} & \multicolumn{2}{|c|}{$\begin{array}{c}\text { Nigeria } \\
(n 13823)\end{array}$} & \multicolumn{2}{|c|}{$\begin{array}{c}\text { Kenya } \\
(n \text { 4623) }\end{array}$} & \multicolumn{2}{|c|}{$\begin{array}{c}\text { Mozambique } \\
(n 7025)\end{array}$} & \multicolumn{2}{|c|}{$\begin{array}{c}\text { DRC } \\
(n 4747) \\
\end{array}$} \\
\hline & Mean or \% & SD & Mean or $\%$ & SD & Mean or \% & SD & Mean or $\%$ & SD & Mean or $\%$ & SD \\
\hline \multicolumn{11}{|l|}{ Dependent variable } \\
\hline $\begin{array}{l}\text { HAZ } \\
\text { Child characteristics }\end{array}$ & $-1 \cdot 01$ & 1.30 & $-1 \cdot 35$ & 1.96 & $-1 \cdot 22$ & 1.43 & $-1 \cdot 66$ & 1.61 & $-1 \cdot 71$ & 1.81 \\
\hline DD score & $2 \cdot 16$ & 1.65 & 1.92 & 1.74 & 2.51 & 1.79 & $2 \cdot 61$ & 1.97 & 1.95 & 1.49 \\
\hline Child's sex (\% female) & 48.49 & - & $49 \cdot 40$ & - & $49 \cdot 00$ & - & 51.00 & - & $50 \cdot 49$ & - \\
\hline Child's age (months) & 25.69 & $15 \cdot 95$ & 26.96 & $16 \cdot 30$ & $25 \cdot 58$ & $15 \cdot 69$ & $27 \cdot 85$ & $15 \cdot 08$ & $28 \cdot 15$ & $16 \cdot 25$ \\
\hline \multicolumn{11}{|l|}{ Maternal characteristics } \\
\hline Mother's education (years) & 5.05 & 4.86 & $5 \cdot 14$ & $5 \cdot 65$ & 6.02 & 4.38 & 3.26 & $3 \cdot 36$ & 4.88 & 3.74 \\
\hline Mother's age (years) & 29.97 & 6.57 & $28 \cdot 77$ & 6.45 & $27 \cdot 88$ & $6 \cdot 13$ & $28 \cdot 29$ & 6.97 & 28.64 & $6 \cdot 43$ \\
\hline Mother's parity & 3.73 & $2 \cdot 10$ & $4 \cdot 27$ & 2.50 & 3.89 & $2 \cdot 36$ & 3.85 & $2 \cdot 26$ & 4.44 & $2 \cdot 46$ \\
\hline Mother working (\% yes) & $76 \cdot 66$ & - & $69 \cdot 27$ & - & $53 \cdot 14$ & - & $37 \cdot 22$ & - & $76 \cdot 64$ & - \\
\hline Mother's BMI $\left(\mathrm{kg} / \mathrm{m}^{2}\right)$ & 23.90 & 4.61 & $22 \cdot 84$ & $4 \cdot 13$ & 22.49 & $4 \cdot 16$ & $22 \cdot 28$ & 3.07 & 21.59 & 3.21 \\
\hline Mother's height $(\mathrm{cm})$ & $15 \cdot 91$ & 0.60 & $15 \cdot 83$ & 0.61 & 15.99 & 0.62 & $15 \cdot 53$ & 0.60 & $15 \cdot 68$ & 7.03 \\
\hline \multicolumn{11}{|l|}{ Household characteristics } \\
\hline \multicolumn{11}{|l|}{ Household wealth index category (\%) } \\
\hline Poorest & 34.65 & - & 21.33 & - & $40 \cdot 42$ & - & $19 \cdot 13$ & - & $27 \cdot 41$ & - \\
\hline Poor & $21 \cdot 61$ & - & 22.90 & - & $20 \cdot 83$ & - & $20 \cdot 51$ & - & $22 \cdot 76$ & - \\
\hline Middle & 18.52 & - & 19.98 & - & $15 \cdot 01$ & - & $20 \cdot 60$ & - & $20 \cdot 16$ & - \\
\hline Rich & 13.54 & - & $19 \cdot 32$ & - & 12.98 & - & 21.61 & - & 17.43 & - \\
\hline Richest & 11.67 & - & $16 \cdot 46$ & - & $10 \cdot 75$ & - & $18 \cdot 15$ & - & $12 \cdot 22$ & - \\
\hline Household size & 6.03 & 2.83 & $7 \cdot 17$ & 3.54 & 6.07 & $2 \cdot 39$ & 6.34 & $2 \cdot 78$ & 6.94 & 2.79 \\
\hline \multicolumn{11}{|l|}{ Child DD components } \\
\hline Grains, roots and tubers (\% yes) & $64 \cdot 6$ & - & $55 \cdot 7$ & - & $62 \cdot 6$ & - & $66 \cdot 1$ & - & $47 \cdot 6$ & - \\
\hline Legumes and nuts (\% yes) & $10 \cdot 8$ & - & $18 \cdot 2$ & - & $16 \cdot 5$ & - & $22 \cdot 9$ & - & $8 \cdot 1$ & - \\
\hline Dairy products (\% yes) & 13.9 & - & $20 \cdot 1$ & - & $48 \cdot 0$ & - & $10 \cdot 2$ & - & $16 \cdot 0$ & - \\
\hline Flesh foods and organ meats (\% yes) & 34.8 & - & $22 \cdot 6$ & - & $15 \cdot 1$ & - & 33.9 & - & 33.8 & - \\
\hline Vitamin-A rich fruits and vegetables ( $\%$ yes) & 28.5 & - & $24 \cdot 0$ & - & 37.5 & - & $46 \cdot 8$ & - & $46 \cdot 4$ & - \\
\hline Eggs (\% yes) & $12 \cdot 4$ & - & $10 \cdot 0$ & - & 11.9 & - & $15 \cdot 3$ & - & $6 \cdot 3$ & - \\
\hline Other fruits and vegetables (\% yes) & $15 \cdot 5$ & - & $15 \cdot 2$ & - & $26 \cdot 9$ & - & 37.5 & - & $20 \cdot 3$ & - \\
\hline
\end{tabular}

DRC; Democratic Republic of Congo; HAZ, height-for-age Z-score; DD, dietary diversity. 
Table 2 Multivariate quantile regression analysis of the association between dietary diversity (DD) and maternal characteristics and child height-for-age Z-score, Ghana, 2014

\begin{tabular}{|c|c|c|c|c|c|c|c|c|c|c|c|c|c|c|}
\hline \multirow[b]{3}{*}{ Variable } & \multirow{2}{*}{\multicolumn{2}{|c|}{ Ordinary least squares regression }} & \multicolumn{12}{|c|}{ Quantile regression } \\
\hline & & & \multicolumn{2}{|c|}{ 5th quantile } & \multicolumn{2}{|c|}{ 10th quantile } & \multicolumn{2}{|c|}{ 25th quantile } & \multicolumn{2}{|c|}{ 50th quantile } & \multicolumn{2}{|c|}{ 75th quantile } & \multicolumn{2}{|c|}{ 90th quantile } \\
\hline & Estimate & SE & Estimate & $\mathrm{SE}$ & Estimate & SE & Estimate & $\mathrm{SE}$ & Estimate & SE & Estimate & SE & Estimate & SE \\
\hline \multicolumn{15}{|l|}{ Child characteristics } \\
\hline DD score & -0.009 & 0.020 & -0.066 & 0.056 & 0.024 & 0.036 & 0.008 & 0.029 & -0.005 & 0.022 & -0.020 & 0.024 & 0.016 & 0.031 \\
\hline Child's sex (female) & $0 \cdot 137^{\star}$ & 0.064 & 0.099 & 0.180 & -0.025 & 0.113 & 0.108 & 0.092 & $0.170^{\star}$ & 0.070 & 0.112 & 0.076 & 0.099 & 0.099 \\
\hline Child's age (months) & $-0.015^{\star \star \star}$ & 0.002 & $-0.022^{\star \star \star}$ & 0.006 & $-0.014^{\star \star \star}$ & 0.004 & $-0.010^{\star *}$ & 0.003 & $-0.011^{\star \star \star}$ & 0.002 & $-0.016^{\star \star \star}$ & 0.002 & $-0.022^{\star \star \star}$ & 0.003 \\
\hline \multicolumn{15}{|l|}{ Maternal characteristics } \\
\hline Mother's education (years) & $0.029^{\star \star *}$ & 0.009 & $0.061^{*}$ & 0.024 & $0.056^{\star \star *}$ & 0.015 & $0.056^{\star * *}$ & 0.012 & 0.018 & 0.009 & 0.016 & 0.010 & $0.034^{*}$ & 0.013 \\
\hline Mother's age (years) & $0.023^{\star \star}$ & 0.007 & 0.005 & 0.021 & 0.005 & 0.013 & $0.024^{*}$ & 0.011 & $0.037^{\star \star \star}$ & 0.008 & $0.027^{\star \star}$ & 0.009 & 0.022 & 0.012 \\
\hline Mother's parity & $-0.078^{\star *}$ & 0.026 & -0.032 & 0.072 & -0.028 & 0.045 & -0.068 & 0.037 & $-0.127^{\star \star \star}$ & 0.028 & $-0 \cdot 110^{\star \star \star}$ & 0.030 & -0.077 & 0.039 \\
\hline Mother working (yes) & -0.097 & 0.079 & -0.068 & 0.222 & 0.002 & 0.140 & -0.087 & 0.113 & -0.108 & 0.087 & -0.064 & 0.094 & -0.192 & 0.122 \\
\hline Mother's BMI $\left(\mathrm{kg} / \mathrm{m}^{2}\right)$ & $0.054^{\star \star \star}$ & 0.008 & $0.060^{\star \star}$ & 0.022 & $0.064^{\star \star \star}$ & 0.014 & $0.056^{\star \star \star}$ & 0.012 & $0.042^{\star \star \star}$ & 0.009 & $0.053^{\star \star \star}$ & 0.009 & $0.068^{\star \star \star}$ & 0.012 \\
\hline Mother's height (cm) & $0.423^{\star \star \star}$ & 0.053 & $0.409^{\star *}$ & $0 \cdot 150$ & $0.356^{\star \star *}$ & 0.095 & $0.465^{\star \star \star}$ & 0.077 & $0.523^{\star \star \star}$ & 0.059 & $0.431^{\star * *}$ & 0.063 & $0.369^{\star * *}$ & 0.082 \\
\hline & & & & & & & & & & & & & & \\
\hline Household wealth index ca & gory & & & & & & & & & & & & & \\
\hline \multicolumn{15}{|c|}{ Poorest (reference) } \\
\hline Wealth index (poor) & $-0 \cdot 131$ & 0.093 & -0.242 & 0.261 & $-0.433^{\star \star}$ & $0 \cdot 164$ & $-0.279^{*}$ & 0.133 & $-0 \cdot 130$ & 0.103 & -0.034 & $0 \cdot 110$ & 0.136 & 0.143 \\
\hline Wealth index (middle) & -0.113 & 0.103 & 0.026 & 0.291 & -0.271 & 0.183 & $-0.292^{*}$ & 0.149 & -0.041 & 0.114 & $-0 \cdot 101$ & 0.123 & -0.110 & 0.160 \\
\hline Wealth index (rich) & -0.027 & 0.120 & -0.021 & 0.337 & -0.239 & 0.212 & -0.239 & 0.172 & -0.039 & 0.133 & 0.062 & 0.142 & 0.103 & 0.185 \\
\hline Wealth index (richest) & 0.073 & 0.145 & 0.173 & 0.407 & -0.193 & 0.256 & -0.180 & 0.208 & 0.142 & 0.160 & 0.193 & 0.172 & 0.032 & 0.224 \\
\hline Household size & -0.008 & 0.013 & 0.030 & 0.036 & -0.008 & 0.023 & -0.006 & 0.019 & -0.001 & 0.014 & -0.009 & 0.015 & -0.004 & 0.020 \\
\hline Observations $(n)$ & \multicolumn{2}{|c|}{1388} & \multicolumn{2}{|c|}{1388} & \multicolumn{2}{|c|}{1388} & \multicolumn{2}{|c|}{1388} & \multicolumn{2}{|c|}{1388} & \multicolumn{2}{|c|}{1388} & \multicolumn{2}{|c|}{1388} \\
\hline
\end{tabular}

${ }^{*} P<0.05,{ }^{* \star} P<0.01,{ }^{* \star *} P<0.001$. 
Table 3 Multivariate quantile regression analysis of the association between dietary diversity (DD) and maternal characteristics and child height-for-age Z-score, Nigeria, 2013

\begin{tabular}{|c|c|c|c|c|c|c|c|c|c|c|c|c|c|c|}
\hline \multirow[b]{3}{*}{ Variable } & \multirow{2}{*}{\multicolumn{2}{|c|}{ Ordinary least squares regression }} & \multicolumn{12}{|c|}{ Quantile regression } \\
\hline & & & \multicolumn{2}{|c|}{ 5th quantile } & \multicolumn{2}{|c|}{ 10th quantile } & \multicolumn{2}{|c|}{ 25th quantile } & \multicolumn{2}{|c|}{ 50th quantile } & \multicolumn{2}{|c|}{ 75th quantile } & \multicolumn{2}{|c|}{ 90th quantile } \\
\hline & Estimate & SE & Estimate & SE & Estimate & SE & Estimate & SE & Estimate & SE & Estimate & SE & Estimate & SE \\
\hline \multicolumn{15}{|l|}{ Child characteristics } \\
\hline DD score & $0.036^{\star \star \star}$ & -0.009 & $0.052^{\star *}$ & -0.019 & $0.046^{\star \star}$ & -0.016 & $0.034^{\star \star}$ & -0.012 & $0.023^{*}$ & -0.010 & 0.021 & -0.012 & $0.065^{\star \star \star}$ & -0.018 \\
\hline Child's sex (female) & $0.219^{\star \star \star}$ & -0.031 & $0 \cdot 291^{\star \star \star}$ & -0.066 & $0.270^{\star \star \star}$ & -0.054 & $0 \cdot 261^{\star \star \star}$ & -0.040 & $0 \cdot 200^{\star \star \star}$ & -0.034 & $0 \cdot 185^{\star \star *}$ & -0.040 & $0 \cdot 179^{\star \star}$ & -0.057 \\
\hline Child's age (months) & $-0.020^{\star * *}$ & -0.001 & -0.001 & -0.002 & $-0.006^{\star \star \star}$ & -0.001 & $-0.012^{\star \star \star}$ & -0.001 & $-0.018^{\star \star *}$ & -0.001 & $-0.026^{\star \star *}$ & -0.001 & $-0.033^{\star \star \star}$ & -0.001 \\
\hline \multicolumn{15}{|l|}{ Maternal characteristics } \\
\hline Mother's education (years) & $0.041^{\star \star *}$ & -0.004 & $0.043^{\star \star \star}$ & -0.008 & $0.057^{\star \star \star}$ & -0.007 & $0.058^{\star \star \star}$ & -0.005 & $0.044^{\star \star \star}$ & -0.004 & $0.030^{\star \star \star}$ & -0.005 & $0.018^{*}$ & -0.007 \\
\hline Mother's age (years) & $0.023^{\star \star *}$ & -0.004 & $0.027^{\star \star *}$ & -0.008 & $0.028^{\star * *}$ & -0.007 & $0.028^{\star \star *}$ & -0.005 & $0.027^{\star \star *}$ & -0.004 & $0.019^{\star \star *}$ & -0.005 & 0.013 & -0.007 \\
\hline Mother's parity & $-0.060^{\star \star \star}$ & -0.010 & $-0.091^{\star \star \star}$ & -0.022 & $-0.070^{\star \star \star}$ & -0.018 & $-0.077^{\star \star \star}$ & -0.014 & $-0.073^{\star \star \star}$ & -0.012 & $-0.050^{\star \star \star}$ & -0.014 & -0.021 & -0.020 \\
\hline Mother working (yes) & $0.074^{*}$ & -0.034 & $0 \cdot 205^{\star \star}$ & -0.073 & 0.112 & -0.059 & $0 \cdot 132^{\star \star}$ & -0.044 & 0.070 & -0.038 & -0.015 & -0.044 & -0.002 & -0.063 \\
\hline Mother's BMI $\left(\mathrm{kg} / \mathrm{m}^{2}\right)$ & $0.034^{\star \star *}$ & -0.004 & $0.031^{* \star *}$ & -0.009 & $0.028^{* * *}$ & -0.007 & $0.031^{* * *}$ & -0.005 & $0.035^{\star \star *}$ & -0.005 & $0.039^{\star \star *}$ & -0.005 & $0.044^{\star \star *}$ & -0.008 \\
\hline Mother's height (cm) & $0.447^{\star \star \star}$ & -0.026 & $0.366^{\star \star \star}$ & -0.055 & $0.445^{\star \star \star}$ & -0.045 & $0.463^{\star \star \star}$ & -0.034 & $0.472^{\star \star \star}$ & -0.029 & $0.464^{\star \star \star}$ & -0.034 & $0.418^{\star \star \star}$ & -0.048 \\
\hline \multirow{2}{*}{\multicolumn{15}{|c|}{$\begin{array}{l}\text { Household characteristics } \\
\text { Household wealth index category } \\
\text { Poorest (reference) }\end{array}$}} \\
\hline & & & & & & & & & & & & & & \\
\hline Wealth index (poor) & $0.235^{\star \star \star}$ & -0.047 & $0 \cdot 131$ & -0.099 & $0.299^{* * *}$ & -0.082 & $0 \cdot 261^{\star \star *}$ & -0.061 & $0.254^{\star \star *}$ & -0.052 & $0.176^{\star \star}$ & -0.062 & $0 \cdot 269^{\star \star}$ & -0.087 \\
\hline Wealth index (middle) & $0.439^{\star \star \star}$ & -0.051 & $0.488^{\star \star \star}$ & $-0 \cdot 108$ & $0.587^{\star \star \star}$ & -0.089 & $0.527^{\star \star \star}$ & -0.066 & $0.508^{\star \star \star}$ & -0.056 & $0.371^{\star \star *}$ & -0.067 & $0.326^{\star \star \star}$ & -0.094 \\
\hline Wealth index (rich) & $0.591^{\star \star \star}$ & -0.055 & $0.681^{\star \star \star}$ & -0.118 & $0.850^{\star \star \star}$ & -0.097 & $0.751^{\star \star \star}$ & -0.072 & $0.652^{\star \star \star}$ & -0.061 & $0.459^{\star \star \star}$ & -0.073 & $0.439^{\star \star \star}$ & -0.103 \\
\hline Wealth index (richest) & $0.696^{\star \star *}$ & -0.066 & $0.787^{\star \star \star}$ & -0.141 & $1 \cdot 019^{* \star \star}$ & -0.116 & $0.845^{\star \star \star}$ & -0.086 & $0.727^{\star \star \star}$ & -0.073 & $0.541^{\star * *}$ & -0.087 & $0.554^{\star \star \star}$ & -0.123 \\
\hline Household size & $-0.013^{\star \star}$ & -0.005 & -0.011 & -0.011 & $-0.022^{*}$ & -0.009 & -0.011 & -0.006 & $-0 \cdot 014^{\star \star}$ & -0.005 & -0.011 & -0.007 & $-0.020^{*}$ & -0.009 \\
\hline Observations (n) & \multicolumn{2}{|c|}{13823} & \multicolumn{2}{|c|}{13823} & \multicolumn{2}{|c|}{13823} & \multicolumn{2}{|c|}{13823} & \multicolumn{2}{|c|}{13823} & \multicolumn{2}{|c|}{13823} & \multicolumn{2}{|c|}{13823} \\
\hline
\end{tabular}

${ }^{\star} P<0.05,{ }^{\star \star} P<0.01,{ }^{* \star \star} P<0.001$. 
Table 4 Multivariate quantile regression analysis of the association between dietary diversity (DD) and maternal characteristics and child height-for-age Z-score (24-59 months), Nigeria, 2013

\begin{tabular}{|c|c|c|c|c|c|c|c|c|c|c|c|c|c|c|}
\hline \multirow[b]{3}{*}{ Variable } & \multirow{2}{*}{\multicolumn{2}{|c|}{ Ordinary least squares regression }} & \multicolumn{12}{|c|}{ Quantile regression } \\
\hline & & & \multicolumn{2}{|c|}{ 5th quantile } & \multicolumn{2}{|c|}{ 10th quantile } & \multicolumn{2}{|c|}{ 25th quantile } & \multicolumn{2}{|c|}{ 50th quantile } & \multicolumn{2}{|c|}{ 75th quantile } & \multicolumn{2}{|c|}{ 90th quantile } \\
\hline & Estimate & SE & Estimate & SE & Estimate & SE & Estimate & SE & Estimate & SE & Estimate & SE & Estimate & SE \\
\hline \multicolumn{15}{|l|}{ Child characteristics } \\
\hline DD score & $0.089^{\star \star *}$ & 0.012 & $0.120^{\star \star *}$ & 0.028 & $0.124^{\star \star *}$ & 0.023 & $0.089^{\star \star \star}$ & 0.016 & $0.049^{\star \star \star}$ & 0.013 & $0.063^{\star \star \star}$ & 0.017 & $0 \cdot 105^{\star \star \star}$ & 0.021 \\
\hline \multirow{2}{*}{\multicolumn{15}{|c|}{ Maternal characteristics }} \\
\hline & & & & & & & & & & & & & & \\
\hline Mother's education (years) & $0.025^{\star \star \star}$ & 0.005 & $0.029^{\star \star}$ & 0.011 & $0.035^{\star \star \star}$ & 0.009 & $0.038^{\star \star \star}$ & 0.006 & $0.034^{\star \star \star}$ & 0.005 & 0.012 & 0.007 & 0.016 & 0.008 \\
\hline Mother's age (years) & $0.024^{\star \star \star}$ & 0.005 & $0.036^{\star \star}$ & 0.012 & $0.040^{\star \star \star}$ & 0.010 & $0.038^{\star \star \star}$ & 0.007 & $0.030^{\star \star \star}$ & 0.006 & $0.019^{\star}$ & 0.007 & 0.012 & 0.009 \\
\hline Mother's parity & $-0.067^{\star \star \star}$ & 0.014 & $-0.113^{\star \star *}$ & 0.033 & $-0 \cdot 105^{\star \star \star}$ & 0.027 & $-0.090^{\star \star \star}$ & 0.019 & $-0.069^{\star \star \star}$ & 0.016 & $-0.067^{\star \star \star}$ & 0.020 & -0.032 & 0.025 \\
\hline Mother working (yes) & 0.023 & 0.045 & 0.157 & 0.104 & 0.157 & 0.085 & 0.082 & 0.060 & 0.056 & 0.050 & -0.005 & 0.064 & $-0.170^{\star}$ & 0.079 \\
\hline Mother's BMI $\left(\mathrm{kg} / \mathrm{m}^{2}\right)$ & $0.047^{\star \star \star}$ & 0.006 & $0.034^{\star \star}$ & 0.013 & $0.033^{\star \star}$ & 0.010 & $0.045^{\star \star \star}$ & 0.007 & $0.043^{\star \star \star}$ & 0.006 & $0.052^{\star \star \star}$ & 0.008 & $0.056^{\star \star \star}$ & 0.010 \\
\hline Mother's height $(\mathrm{cm})$ & $0.446^{\star * *}$ & 0.035 & $0.301^{\star * *}$ & 0.080 & $0.382^{\star \star \star}$ & 0.065 & $0.433^{\star \star \star}$ & 0.046 & $0.445^{\star \star \star}$ & 0.038 & $0.518^{\star \star \star}$ & 0.049 & $0.446^{\star \star \star}$ & 0.061 \\
\hline \multirow{2}{*}{\multicolumn{15}{|c|}{$\begin{array}{l}\text { Household characteristics } \\
\text { Household wealth index category } \\
\text { Poorest (reference) }\end{array}$}} \\
\hline & & & & & & & & & & & & & & \\
\hline Wealth index (poor) & $0.239^{\star \star \star}$ & 0.061 & 0.118 & 0.140 & $0.315^{\star \star}$ & 0.114 & $0.393^{\star \star \star}$ & 0.080 & $0.244^{\star \star \star}$ & 0.067 & 0.157 & 0.086 & $0.234^{\star}$ & 0.106 \\
\hline Wealth index (middle) & $0.468^{\star * *}$ & 0.066 & $0.609^{\star * \star}$ & 0.152 & $0.594^{* \star *}$ & 0.124 & $0.689^{\star \star \star}$ & 0.087 & $0.492^{* \star \star}$ & 0.073 & $0.368^{\star * *}$ & 0.094 & $0.338^{* *}$ & 0.116 \\
\hline Wealth index (rich) & $0.652^{\star \star \star}$ & 0.073 & $0.898^{\star * *}$ & 0.167 & $0.931^{\star \star *}$ & 0.137 & $0.937^{\star \star \star}$ & 0.096 & $0.711^{\star \star \star}$ & 0.080 & $0.496^{\star \star *}$ & 0.103 & $0.264^{\star}$ & 0.127 \\
\hline Wealth index (richest) & $0.897^{\star \star \star}$ & 0.088 & $1 \cdot 192^{\star \star \star}$ & 0.201 & $1.338^{\star \star \star}$ & 0.164 & $1 \cdot 217^{\star \star \star}$ & 0.115 & $0.904^{\star \star \star}$ & 0.096 & $0.692^{\star \star \star}$ & 0.124 & $0.450^{\star \star}$ & 0.153 \\
\hline Household size & $-0 \cdot 015^{\star}$ & 0.007 & -0.016 & 0.015 & -0.013 & 0.013 & $-0.022^{*}$ & 0.009 & $-0.022^{\star \star}$ & 0.007 & -0.011 & 0.009 & -0.019 & 0.012 \\
\hline Observations $(n)$ & \multicolumn{2}{|c|}{6166} & \multicolumn{2}{|c|}{6166} & \multicolumn{2}{|c|}{6166} & \multicolumn{2}{|c|}{6166} & \multicolumn{2}{|c|}{6166} & \multicolumn{2}{|c|}{6166} & \multicolumn{2}{|c|}{6166} \\
\hline
\end{tabular}

${ }^{\star} P<0.05,{ }^{* \star} P<0.01,{ }^{* \star *} P<0.001$. 
Table 5 Multivariate quantile regression analysis of the association between dietary diversity (DD) and maternal characteristics and child height-for-age Z-score (6-23 months), Nigeria, 2013

\begin{tabular}{|c|c|c|c|c|c|c|c|c|c|c|c|c|c|c|}
\hline \multirow[b]{3}{*}{ Variable } & \multirow{2}{*}{\multicolumn{2}{|c|}{ Ordinary least squares regression }} & \multicolumn{12}{|c|}{ Quantile regression } \\
\hline & & & \multicolumn{2}{|c|}{ 5th quantile } & \multicolumn{2}{|c|}{ 10th quantile } & \multicolumn{2}{|c|}{ 25th quantile } & \multicolumn{2}{|c|}{ 50th quantile } & \multicolumn{2}{|c|}{ 75th quantile } & \multicolumn{2}{|c|}{ 90th quantile } \\
\hline & Estimate & SE & Estimate & SE & Estimate & SE & Estimate & SE & Estimate & SE & Estimate & SE & Estimate & SE \\
\hline \multicolumn{15}{|l|}{ Child characteristics } \\
\hline DD score & $-0.029^{\star}$ & 0.013 & -0.007 & 0.027 & -0.026 & 0.022 & -0.005 & 0.016 & $-0.031^{*}$ & 0.015 & $-0.046^{\star}$ & 0.018 & -0.029 & 0.027 \\
\hline Child's sex (female) & $0.325^{\star \star \star}$ & 0.045 & $0.397^{* \star \star}$ & 0.091 & $0.400^{\star \star \star}$ & 0.074 & $0.427^{\star \star \star}$ & 0.053 & $0 \cdot 297^{\star \star \star}$ & 0.050 & $0.339^{\star \star \star}$ & 0.060 & $0.282^{\star \star}$ & 0.088 \\
\hline \multicolumn{15}{|l|}{ Maternal characteristics } \\
\hline Mother's education (years) & $0.059^{\star \star *}$ & 0.006 & $0.068^{\star \star \star}$ & 0.012 & $0.069^{\star \star *}$ & 0.009 & $0.076^{\star * *}$ & 0.007 & $0.061^{\star \star *}$ & 0.006 & $0.053^{\star \star *}$ & 0.008 & $0.031^{\star \star}$ & 0.011 \\
\hline Mother's age (years) & $0 \cdot 011^{\star \star \star}$ & 0.005 & $0 \cdot 025^{\star}$ & 0.011 & $0.023^{\star \star}$ & 0.009 & $0.016^{*}$ & 0.006 & $0.024^{\star \star *}$ & 0.006 & $0 \cdot 018^{\star}$ & 0.007 & 0.007 & 0.011 \\
\hline Mother's parity & $-0.058^{\star \star *}$ & 0.015 & $-0.077^{\star}$ & 0.031 & $-0.070^{\star *}$ & 0.025 & $-0.059^{\star *}$ & 0.018 & $-0.081^{\star * *}$ & 0.017 & $-0.047^{*}$ & 0.021 & -0.013 & 0.030 \\
\hline Mother working (yes) & 0.097 & 0.049 & $0.233^{*}$ & $0 \cdot 101$ & 0.108 & 0.082 & $0.149^{\star}$ & 0.059 & 0.106 & 0.056 & -0.044 & 0.067 & 0.058 & 0.098 \\
\hline Mother's BMI $\left(\mathrm{kg} / \mathrm{m}^{2}\right)$ & $0.024^{\star * *}$ & 0.006 & $0.024^{*}$ & 0.012 & $0.028^{* *}$ & 0.010 & $0.019^{\star *}$ & 0.007 & $0.022^{\star \star \star}$ & 0.007 & $0.026^{\star \star}$ & 0.008 & $0.029^{*}$ & 0.012 \\
\hline Mother's height (cm) & $0.447^{\star \star \star}$ & 0.037 & $0.388^{\star \star *}$ & 0.076 & $0.504^{\star \star *}$ & 0.062 & $0.491^{* * *}$ & 0.044 & $0.491^{* * *}$ & 0.042 & $0.440^{\star \star *}$ & 0.050 & $0.447^{\star \star *}$ & 0.073 \\
\hline \multicolumn{15}{|c|}{ Household characteristics } \\
\hline \multicolumn{15}{|c|}{$\begin{array}{l}\text { Household wealth index category } \\
\text { Poorest (reference) }\end{array}$} \\
\hline Wealth index (poor) & $0.230^{\star \star \star}$ & 0.069 & 0.207 & $0 \cdot 141$ & 0.221 & $0 \cdot 114$ & $0.183^{*}$ & 0.082 & $0.267^{\star \star \star}$ & 0.078 & $0 \cdot 253^{\star *}$ & 0.093 & $0.302^{*}$ & 0.137 \\
\hline Wealth index (middle) & $0.400^{\star * *}$ & 0.075 & $0.481^{* *}$ & 0.153 & $0.499^{\star \star \star}$ & 0.124 & $0.408^{* * *}$ & 0.089 & $0.451^{\star \star *}$ & 0.084 & $0.419^{\star \star \star}$ & 0.101 & 0.228 & 0.148 \\
\hline Wealth index (rich) & $0.523^{\star * *}$ & 0.082 & $0.635^{\star \star \star}$ & 0.166 & $0.681^{* \star *}$ & 0.135 & $0.675^{\star \star \star}$ & 0.097 & $0.521^{* \star *}$ & 0.092 & $0.428^{\star \star \star}$ & 0.110 & $0.511^{\star \star}$ & 0.161 \\
\hline Wealth index (richest) & $0.532^{\star \star \star}$ & 0.097 & $0.533^{\star \star}$ & 0.197 & $0.798^{\star \star \star}$ & 0.160 & $0.634^{\star \star \star}$ & 0.115 & $0.505^{\star \star \star}$ & 0.109 & $0.463^{\star \star \star}$ & 0.130 & $0.521^{\star \star}$ & 0.191 \\
\hline Household size & -0.011 & 0.007 & -0.016 & 0.014 & -0.019 & 0.012 & -0.006 & 0.008 & -0.011 & 0.008 & -0.004 & 0.010 & -0.012 & 0.014 \\
\hline Observations ( $n$ ) & & & 7657 & & 7657 & & 7657 & & 7657 & & 7657 & & 7657 & \\
\hline
\end{tabular}


Table 6 Multivariate quantile regression analysis of the association between dietary diversity (DD) and maternal characteristics and child height-for-age Z-score, Kenya, 2014

\begin{tabular}{|c|c|c|c|c|c|c|c|c|c|c|c|c|c|c|}
\hline \multirow[b]{3}{*}{ Variable } & \multirow{2}{*}{\multicolumn{2}{|c|}{ Ordinary least squares regression }} & \multicolumn{12}{|c|}{ Quantile regression } \\
\hline & & & \multicolumn{2}{|c|}{ 5th quantile } & \multicolumn{2}{|c|}{ 10th quantile } & \multicolumn{2}{|c|}{ 25th quantile } & \multicolumn{2}{|c|}{ 50th quantile } & \multicolumn{2}{|c|}{ 75th quantile } & \multicolumn{2}{|c|}{ 90th quantile } \\
\hline & Estimate & SE & Estimate & SE & Estimate & SE & Estimate & SE & Estimate & SE & Estimate & SE & Estimate & SE \\
\hline \multicolumn{15}{|l|}{ Child characteristics } \\
\hline DD score & -0.005 & 0.012 & 0.027 & 0.029 & 0.008 & 0.021 & -0.019 & 0.014 & -0.020 & 0.013 & -0.016 & 0.015 & 0.004 & 0.021 \\
\hline Child's sex (female) & $0.233^{\star \star \star}$ & 0.039 & $0.227^{*}$ & 0.094 & $0.324^{\star \star \star}$ & 0.070 & $0.277^{\star \star \star}$ & 0.046 & $0.234^{\star \star \star}$ & 0.043 & $0 \cdot 154^{\star \star}$ & 0.049 & $0 \cdot 160^{\star}$ & 0.069 \\
\hline Child's age (months) & $-0.013^{\star \star \star}$ & 0.001 & -0.005 & 0.003 & $-0.006^{\star}$ & 0.002 & $-0.007^{\star \star *}$ & 0.001 & $-0.010^{* * *}$ & 0.001 & $-0.016^{\star \star *}$ & 0.001 & $-0.018^{\star \star \star}$ & 0.002 \\
\hline \multicolumn{15}{|l|}{ Maternal characteristics } \\
\hline Mother's education (years) & $-0.025^{\star \star \star}$ & 0.006 & 0.003 & 0.015 & 0.001 & 0.011 & -0.010 & 0.007 & $-0.019^{\star *}$ & 0.007 & $-0.026^{\star \star \star}$ & 0.008 & $-0.044^{\star \star \star}$ & 0.011 \\
\hline Mother's age (years) & $0.011^{*}$ & 0.005 & 0.008 & 0.012 & 0.014 & 0.009 & $0.017^{\star \star}$ & 0.006 & $0.013^{\star}$ & 0.006 & 0.009 & 0.006 & -0.0004 & 0.009 \\
\hline Mother's parity & $-0.049^{\star \star}$ & 0.016 & -0.065 & 0.038 & $-0.068^{\star}$ & 0.028 & $-0.082^{\star \star \star}$ & 0.018 & $-0.058^{\star \star \star}$ & 0.018 & $-0.040^{\star}$ & 0.020 & 0.016 & 0.027 \\
\hline Mother working (yes) & $-0 \cdot 181^{\star \star \star}$ & 0.043 & $-0.235^{\star}$ & 0.102 & $-0.194^{\star *}$ & 0.075 & -0.084 & 0.049 & $-0 \cdot 178^{\star \star \star}$ & 0.047 & $-0.269^{\star \star \star}$ & 0.053 & $-0.234^{\star *}$ & 0.074 \\
\hline Mother's BMI $\left(\mathrm{kg} / \mathrm{m}^{2}\right)$ & $0.035^{\star \star *}$ & 0.005 & $0.033^{*}$ & 0.013 & $0.026^{\star \star}$ & 0.009 & $0.031^{* \star *}$ & 0.006 & $0.037^{\star \star *}$ & 0.006 & $0.037^{\star \star \star}$ & 0.007 & $0.054^{\star * *}$ & 0.009 \\
\hline Mother's height (cm) & $0.500^{* * *}$ & 0.032 & $0.355^{\star \star *}$ & 0.076 & $0.463^{\star \star \star}$ & 0.056 & $0.502^{\star * \star}$ & 0.037 & $0.542^{\star \star \star}$ & 0.035 & $0.542^{* \star *}$ & 0.040 & $0.446^{\star * \star}$ & 0.056 \\
\hline \multirow{3}{*}{\multicolumn{15}{|c|}{$\begin{array}{l}\text { Household characteristics } \\
\text { Household wealth index category } \\
\text { Poorest (reference) }\end{array}$}} \\
\hline & & & & & & & & & & & & & & \\
\hline & & & & & & & & & & & & & & \\
\hline Wealth index (poor) & $0.171^{\star \star}$ & 0.059 & 0.098 & 0.140 & $0.243^{*}$ & 0.104 & $0.159^{*}$ & 0.068 & $0.133^{\star}$ & 0.065 & $0.165^{\star}$ & 0.073 & 0.118 & 0.102 \\
\hline Wealth index (middle) & $0.345^{\star \star \star}$ & 0.066 & 0.256 & 0.159 & $0.329^{\star *}$ & 0.117 & $0.305^{\star \star \star}$ & 0.077 & $0.368^{\star \star \star}$ & 0.073 & $0.354^{\star \star \star}$ & 0.082 & $0.389^{\star \star \star}$ & 0.116 \\
\hline Wealth index (rich) & $0.310^{\star \star \star}$ & 0.072 & -0.015 & 0.173 & 0.131 & 0.128 & $0.292^{\star \star \star}$ & 0.084 & $0.262^{\star \star}$ & 0.080 & $0.398^{\star \star \star}$ & 0.090 & $0.475^{\star \star \star}$ & 0.126 \\
\hline Wealth index (richest) & $0.706^{\star \star \star}$ & 0.085 & 0.204 & 0.204 & $0.437^{\star \star}$ & 0.151 & $0.711^{\star \star \star *}$ & 0.099 & $0.738^{\star \star \star}$ & 0.094 & $0.645^{\star \star *}$ & 0.106 & $0.857^{\star \star \star}$ & 0.149 \\
\hline Household size & \multirow{2}{*}{\multicolumn{2}{|c|}{4623}} & 0.004 & 0.025 & -0.009 & 0.018 & 0.010 & 0.012 & -0.003 & 0.011 & -0.010 & 0.013 & $-0.038^{\star}$ & 0.018 \\
\hline Observations $(n)$ & & & \multicolumn{2}{|c|}{4623} & \multicolumn{2}{|c|}{4623} & \multicolumn{2}{|c|}{4623} & 4623 & & \multicolumn{2}{|c|}{4623} & \multicolumn{2}{|c|}{4623} \\
\hline
\end{tabular}

${ }^{*} P<0.05,{ }^{* *} P<0.01,{ }^{* * *} P<0.001$. 
Table 7 Multivariate quantile regression analysis of the association between dietary diversity (DD) and maternal characteristics and child height-for-age Z-score, Mozambique, 2011

\begin{tabular}{|c|c|c|c|c|c|c|c|c|c|c|c|c|c|c|}
\hline \multirow[b]{3}{*}{ Variable } & \multirow{2}{*}{\multicolumn{2}{|c|}{ Ordinary least squares regression }} & \multicolumn{12}{|c|}{ Quantile regression } \\
\hline & & & \multicolumn{2}{|c|}{ 5th quantile } & \multicolumn{2}{|c|}{ 10th quantile } & \multicolumn{2}{|c|}{ 25th quantile } & \multicolumn{2}{|c|}{ 50th quantile } & \multicolumn{2}{|c|}{ 75th quantile } & \multicolumn{2}{|c|}{ 90th quantile } \\
\hline & Estimate & SE & Estimate & SE & Estimate & SE & Estimate & SE & Estimate & SE & Estimate & SE & Estimate & SE \\
\hline \multicolumn{15}{|l|}{ Child characteristics } \\
\hline DD score & 0.016 & 0.009 & 0.025 & 0.023 & 0.028 & 0.018 & 0.012 & 0.011 & 0.004 & 0.009 & 0.013 & 0.013 & 0.026 & 0.017 \\
\hline Child's sex (female) & $0.208^{* * *}$ & 0.037 & $0.302^{* * *}$ & 0.090 & $0.286^{\star * \star}$ & 0.071 & $0.176^{\star * *}$ & 0.043 & $0.169^{\star * \star}$ & 0.039 & $0 \cdot 170^{\star \star *}$ & 0.050 & $0.154^{\star}$ & 0.069 \\
\hline Child's age (months) & $-0.012^{\star \star *}$ & 0.001 & $0.007^{\star}$ & 0.003 & 0.002 & 0.002 & -0.001 & 0.001 & $-0.006^{\star \star *}$ & 0.001 & $-0 \cdot 015^{\star \star *}$ & 0.002 & $-0.023^{\star \star *}$ & 0.002 \\
\hline \multicolumn{15}{|l|}{ Maternal characteristics } \\
\hline Mother's education (years) & $0.020^{* *}$ & 0.007 & $0.046^{\star \star}$ & 0.018 & $0.039^{\star \star}$ & 0.014 & $0.029^{\star * *}$ & 0.008 & 0.014 & 0.008 & 0.018 & 0.009 & -0.004 & 0.013 \\
\hline Mother's age (years) & $0 \cdot 015^{\star \star \star}$ & 0.004 & 0.009 & 0.010 & 0.016 & 0.008 & $0.013^{\star \star}$ & 0.004 & $0.013^{\star \star}$ & 0.004 & 0.004 & 0.006 & $0.019^{\star}$ & 0.008 \\
\hline Mother's parity & $-0.030^{\star}$ & 0.014 & -0.024 & 0.034 & $-0.056^{\star}$ & 0.027 & -0.025 & 0.016 & -0.020 & 0.015 & 0.003 & 0.019 & -0.050 & 0.026 \\
\hline Mother working (yes) & 0.030 & 0.038 & 0.096 & 0.094 & 0.040 & 0.074 & 0.062 & 0.044 & 0.024 & 0.040 & 0.017 & 0.052 & -0.089 & 0.071 \\
\hline Mother's BMI $\left(\mathrm{kg} / \mathrm{m}^{2}\right)$ & $0.039^{\star * *}$ & 0.006 & 0.004 & 0.016 & $0.024^{\star}$ & 0.012 & $0.028^{\star \star \star}$ & 0.007 & $0.036^{\star * *}$ & 0.007 & $0.055^{\star \star \star}$ & 0.009 & $0.062^{\star \star *}$ & 0.012 \\
\hline Mother's height (cm) & $0.475^{\star \star *}$ & 0.032 & $0.529^{\star \star *}$ & 0.079 & $0.567^{\star \star \star}$ & 0.062 & $0.547^{\star \star \star}$ & 0.037 & $0.544^{\star * *}$ & 0.034 & $0.461^{\star \star *}$ & 0.044 & $0.369^{* \star *}$ & 0.060 \\
\hline \multirow{3}{*}{\multicolumn{15}{|c|}{$\begin{array}{l}\text { Household characteristics } \\
\text { Household wealth index category } \\
\text { Poorest (reference) }\end{array}$}} \\
\hline & & & & & & & & & & & & & & \\
\hline & & & & & & & & & & & & & & \\
\hline Wealth index (poor) & 0.087 & 0.058 & 0.009 & 0.143 & 0.137 & 0.113 & 0.069 & 0.068 & $0.178^{\star \star}$ & 0.062 & 0.097 & 0.080 & -0.102 & 0.109 \\
\hline Wealth index (middle) & 0.115 & 0.059 & 0.053 & 0.145 & 0.087 & 0.114 & $0 \cdot 157^{\star}$ & 0.068 & $0 \cdot 157^{\star}$ & 0.063 & 0.054 & 0.081 & -0.051 & 0.110 \\
\hline Wealth index (rich) & $0 \cdot 158^{\star}$ & 0.061 & $0.360^{\star}$ & 0.151 & $0.279^{\star}$ & 0.119 & $0.305^{\star \star \star}$ & 0.071 & $0.237^{\star \star \star}$ & 0.065 & 0.032 & 0.084 & $-0.232^{*}$ & 0.115 \\
\hline Wealth index (richest) & $0.424^{\star \star \star}$ & 0.075 & $0.691^{\star \star \star}$ & 0.184 & $0.668^{\star \star \star}$ & 0.144 & $0.546^{\star \star \star}$ & 0.087 & $0.530^{\star \star \star}$ & 0.079 & $0.321^{\star \star}$ & 0.102 & 0.141 & 0.140 \\
\hline Household size & $0.023^{\star * *}$ & 0.007 & 0.021 & 0.018 & 0.026 & 0.014 & $0.019^{\star}$ & 0.008 & $0.016^{*}$ & 0.008 & $0.024^{\star}$ & 0.009 & $0.026^{\star}$ & 0.013 \\
\hline Observations $(n)$ & \multicolumn{2}{|c|}{7025} & \multicolumn{2}{|c|}{7025} & \multicolumn{2}{|c|}{7025} & \multicolumn{2}{|c|}{7025} & \multicolumn{2}{|c|}{7025} & \multicolumn{2}{|c|}{7025} & \multicolumn{2}{|c|}{7025} \\
\hline
\end{tabular}


Table 8 Multivariate quantile regression analysis of the association dietary diversity (DD) and maternal characteristics and child height-for-age Z-score, Democratic Republic of Congo, 2013

\begin{tabular}{|c|c|c|c|c|c|c|c|c|c|c|c|c|c|c|}
\hline \multirow[b]{3}{*}{ Variable } & \multirow{2}{*}{\multicolumn{2}{|c|}{ Ordinary least squares regression }} & \multicolumn{12}{|c|}{ Quantile regression } \\
\hline & & & \multicolumn{2}{|c|}{ 5th quantile } & \multicolumn{2}{|c|}{ 10th quantile } & \multicolumn{2}{|c|}{ 25th quantile } & \multicolumn{2}{|c|}{ 50th quantile } & \multicolumn{2}{|c|}{ 75th quantile } & \multicolumn{2}{|c|}{ 90th quantile } \\
\hline & Estimate & SE & Estimate & SE & Estimate & SE & Estimate & SE & Estimate & SE & Estimate & SE & Estimate & SE \\
\hline \multicolumn{15}{|l|}{ Child characteristics } \\
\hline DD score & $0.035^{\star}$ & 0.017 & $0.073^{\star}$ & 0.030 & $0.066^{*}$ & 0.027 & 0.041 & 0.021 & 0.017 & 0.018 & 0.031 & 0.022 & 0.039 & 0.036 \\
\hline Child's sex (female) & $0.321^{\star \star *}$ & 0.048 & $0.261^{\star *}$ & 0.086 & $0.265^{\star \star *}$ & 0.078 & $0.340^{\star \star *}$ & 0.061 & $0.384^{\star \star *}$ & 0.052 & $0.262^{\star \star *}$ & 0.065 & $0.302^{\star \star}$ & 0.104 \\
\hline Child's age (months) & $-0.033^{\star \star \star}$ & 0.002 & $-0.022^{\star \star \star}$ & 0.003 & $-0.026^{\star \star \star}$ & 0.002 & $-0.029^{\star \star \star}$ & 0.002 & $-0.032^{\star \star \star}$ & 0.002 & $-0.034^{\star \star \star}$ & 0.002 & $-0.039^{\star \star \star}$ & 0.003 \\
\hline \multicolumn{15}{|l|}{ Maternal characteristics } \\
\hline Mother's education (years) & $0.024^{\star *}$ & 0.008 & $0.055^{\star \star \star}$ & 0.014 & $0.051^{\star \star *}$ & 0.012 & $0.037^{\star * \star}$ & 0.009 & $0.018^{*}$ & 0.008 & $0.021^{*}$ & 0.010 & -0.005 & 0.017 \\
\hline Mother's age (years) & $0.028^{\star \star \star}$ & 0.006 & $0.030^{\star \star}$ & 0.011 & $0.029^{\star \star}$ & 0.009 & $0.026^{\star \star \star}$ & 0.008 & $0.032^{\star \star \star}$ & 0.007 & $0.034^{\star \star \star}$ & 0.008 & 0.023 & 0.013 \\
\hline Mother's parity & $-0.099^{\star \star \star}$ & 0.017 & $-0.084^{\star *}$ & 0.031 & $-0.086^{\star *}$ & 0.028 & $-0.093^{\star * *}$ & 0.022 & $-0 \cdot 116^{\star \star *}$ & 0.019 & $-0 \cdot 115^{\star * *}$ & 0.023 & $-0 \cdot 104^{\star *}$ & 0.037 \\
\hline Mother working (yes) & -0.045 & 0.058 & 0.064 & $0 \cdot 103$ & -0.043 & 0.094 & -0.050 & 0.074 & -0.090 & 0.063 & -0.114 & 0.078 & 0.065 & 0.126 \\
\hline Mother's BMI $\left(\mathrm{kg} / \mathrm{m}^{2}\right)$ & $0.017^{\star}$ & 0.008 & -0.004 & 0.014 & 0.002 & 0.013 & 0.008 & 0.010 & $0.025^{\star \star}$ & 0.009 & 0.017 & 0.011 & 0.021 & 0.018 \\
\hline Mother's height $(\mathrm{cm})$ & $0.499^{\star * *}$ & 0.035 & $0.452^{\star \star *}$ & 0.063 & $0.438^{\star \star \star}$ & 0.057 & $0.507^{\star \star *}$ & 0.045 & $0.524^{\star \star *}$ & 0.038 & $0.522^{\star \star *}$ & 0.047 & $0.437^{\star \star \star}$ & 0.077 \\
\hline \multirow{2}{*}{\multicolumn{15}{|c|}{$\begin{array}{l}\text { Household characteristics } \\
\text { Household wealth index category }\end{array}$}} \\
\hline & & & & & & & & & & & & & & \\
\hline \multicolumn{15}{|c|}{ Poorest (reference) } \\
\hline Wealth index (poor) & -0.088 & 0.068 & 0.007 & 0.122 & -0.068 & 0.111 & -0.068 & 0.088 & -0.138 & 0.075 & $-0 \cdot 230^{\star}$ & 0.092 & 0.020 & 0.149 \\
\hline Wealth index (middle) & 0.066 & 0.071 & 0.134 & 0.128 & 0.120 & 0.116 & 0.091 & 0.092 & 0.062 & 0.078 & -0.131 & 0.097 & 0.060 & 0.156 \\
\hline Wealth index (rich) & 0.084 & 0.078 & $0 \cdot 138$ & 0.139 & 0.004 & 0.126 & $0 \cdot 146$ & 0.099 & $0 \cdot 158$ & 0.085 & -0.048 & 0.105 & 0.067 & 0.170 \\
\hline Wealth index (richest) & $0.441^{\star \star \star}$ & 0.099 & $0.641^{\star \star \star}$ & 0.179 & $0.574^{\star \star \star}$ & 0.162 & $0.617^{\star \star \star}$ & 0.128 & $0.527^{\star \star \star}$ & 0.109 & $0.296^{*}$ & 0.135 & 0.353 & 0.218 \\
\hline Household size & 0.007 & 0.009 & -0.018 & 0.017 & -0.019 & 0.016 & 0.008 & 0.012 & 0.014 & 0.011 & $0.027^{*}$ & 0.013 & 0.007 & 0.021 \\
\hline Observations $(n)$ & \multicolumn{2}{|c|}{4745} & \multicolumn{2}{|c|}{4745} & \multicolumn{2}{|c|}{4745} & \multicolumn{2}{|c|}{4745} & \multicolumn{2}{|c|}{4745} & \multicolumn{2}{|c|}{4745} & \multicolumn{2}{|c|}{4745} \\
\hline
\end{tabular}

${ }^{*} P<0.05,{ }^{* *} P<0.01,{ }^{* * *} P<0.001$. 
in the two lowest quantiles (5th, 10th), with the larger effect occurring at the 5th quantile. However, the age-specific analysis showed only a positive mean effect (OLS) of DD on HAZ of children aged 24-59 months and no significant effect was observed in the quantile results (results not shown). The effect was not significant in the three remaining countries.

In the analysis of maternal years of education, a decreasing effect on HAZ was observed across quantiles in Ghana, Nigeria and DRC. In these countries, the largest effect of maternal years of education occurred at the lowest quantile (5th) and least effect at the upper quantiles (90th for Ghana and Nigeria, 75th for DRC). In Mozambique, education was significantly associated with HAZ in the first three lower quantiles (5th, 10th, 25th), with the largest effect occurring at the 5 th quantile. No significant effects were observed at the upper quantiles. Conversely, maternal education was negatively associated with child HAZ at the three upper quantiles (50th, 75th and 90th) in Kenya. A 1-year increase in maternal education was associated with a decrease in child HAZ of 0.019, 0.026 and 0.044 at the 50th, 75th and 90th quantile, respectively. No significant effect was observed at the lower end of the HAZ distribution. Maternal work status was significantly and positively associated with child HAZ at 5th and 25th quantiles in Nigeria, with the largest effect being at the 5th quantile. Contrarily, maternal employment had a negative effect on HAZ at the 5th, 10th, 50th, 75th and 90th quantiles in Kenya. Thus, mothers who said they were working and were in the 5th, 10th, 50th, 75th and 90th quantiles tended to have children who had low HAZ. The greatest effects were at the lowest (5th) and the upper quantiles (75th, 90th). There was no effect at the 25th quantile.

Maternal age was significantly and positively associated with at least two quantiles of child HAZ across all countries. Maternal parity had a negative effect on HAZ at two upper quantiles (50th, 75th) in Ghana and one lowest quantile (10th) in Mozambique. This inverse relationship was also found in the 5th, 10th, 25th, 50th and 75th quantiles in Nigeria and Kenya; while in DRC the negative effect was across all quantiles, with the largest effect at the upper quantiles. Parity was not associated significantly with child HAZ at the 90th quantiles. Except in DRC where the positive effect of maternal BMI on HAZ occurred only at the 50th quantile, in the remaining four countries the effect was throughout the conditional HAZ distribution (excluding the 5th quantile in Mozambique) in linear ways - the least effect was at the lowest quantile (5th) and the largest effect at the highest quantile (90th) in the case of Ghana, Nigeria and Kenya. Maternal height had an increasing positive effect across quantiles in Ghana, Nigeria, Kenya and DRC, and peaked at the 50th quantile. An increase of $1 \mathrm{~cm}$ in maternal height was associated with a $0.523,0.472,0.542$ and 0.524 increase in child's HAZ at the 50th quantile in Ghana, Nigeria, Kenya and DRC, respectively. A similar trend was observed in
Mozambique, but with the largest effect occurring at the 10th quantile.

\section{Discussion}

The present paper examined the association between child DD and maternal characteristics and child HAZ in five sub-Saharan African countries: Ghana, Nigeria, Kenya, Mozambique and DRC, using QR to depict the differential effects of each putative correlate of HAZ. Net of controls, the results show that DD has a significant positive effect mostly at the lower end of the conditional HAZ distribution in Nigeria and DRC. In both countries the largest effect occurs at the lowest quantile (5th). This finding suggests that children at the lower tail of the HAZ distribution, who are likely to be at a greater risk of undernutrition, benefit more from improvement in DD than those in the upper end of the distribution. Therefore DD interventions to address child nutrition in these two countries may have more impact on children who are at higher risk of malnutrition. These differential effects of DD on child HAZ are contrary to OLS results, which misleadingly indicate that DD has a significant positive effect on the HAZ of all children in the two countries. Further, stratifying the analysis by age reveals an interesting finding in Nigeria. While the effect of DD on HAZ was positive across all quantiles for children aged 24 months and above and with a greater magnitude than in the combined sample, the effect was negative for children between the ages of 6 and 23 months in two upper quantiles (50th and 75th). This is somewhat surprising, because, although there is evidence in the literature that points to the fact that DD has no effect on the linear growth of children in this age category ${ }^{(46)}$, the inverse relationship is puzzling and will need further investigation to unravel the factors that might have contributed to the negative relationship. In the remaining three countries, DD has no effect on child HAZ in any of the quantiles; therefore suggesting that DD may not be an effective intervention strategy to address child nutrition in these settings. These findings are in line with previous research. A study using DHS data from eleven countries in Africa and Latin America observed a significant positive association between child DD and $H_{A Z}{ }^{(14)}$. Several other studies have observed a positive relationship between DD and child growth ${ }^{(9-11)}$. However, a related study in Ghana did not find a significant association between child DD and linear growth, even though in that study DD was significantly associated with childhood wasting, another indicator of undernutrition ${ }^{(13)}$. The limitation of the above studies is that they all investigated the average effect of DD on child growth. It is therefore significant to point out that the findings of those studies are consistent with OLS results obtained in the present study but only partly consistent with the QR results, as the QR results show differential effects of DD along the conditional HAZ distribution. 
The differences in effect of DD on children's HAZ among countries as illustrated above are not unexpected, as previous researchers using data from multiple countries have shown that the effect of DD is not across all countries $^{(14)}$ - while it is associated significantly with child linear growth in some countries, it is not the case for child growth in others. This could be attributed to different DD consumption patterns among and across countries. This can be seen in the descriptive analysis of the present study, where consumption of the individual food groups varies from country to country. This may lead to different DD scores, as observed in our descriptive analysis, and consequently different effects on child linear growth. Similarly, the differences in effect of DD on child HAZ across quantiles, as illustrated in the Nigerian data, could be due to the fact that children with certain level of HAZ are more or less sensitive to the effect of DD than others. This may be the case because linear growth, which is a biological process and varies from person to person, also responds differently to the environment ${ }^{(47)}$.

Maternal education is another critical variable that influences child linear growth. Our results show that maternal years of education have significant positive effects on child HAZ in Ghana, Nigeria, Mozambique and DRC, with the largest effects being at the lower end of the HAZ distribution. This suggests that the beneficial effects of maternal years of education on HAZ accrue disproportionately to children in the lower tail of the conditional HAZ distribution. The implication of this may be that maternal education interventions to improve child growth may be more impactful for linear growth of children at the lower end of the conditional HAZ distribution and who are also likely to be at greatest risk of undernutrition. However, the OLS results show that maternal education has a strong effect, with HAZ increasing with years of education for all children in the four countries; this paints just a part of the picture and therefore can be misleading. Conversely, maternal education has a negative effect on child HAZ at the upper tail of the conditional HAZ distribution in Kenya. The inverse relationship between maternal education and HAZ is puzzling and further research would be needed to elucidate the factors accounting for this negative relationship. There was, however, no significant effect of maternal education on HAZ of children in the lower end of the distribution. This suggests that policy interventions aiming to increase maternal years of education are unlikely to be effective in improving the HAZ of Kenyan children at the lower end of the conditional HAZ distribution. Relating the results of the present study to what is in the literature, one can observe some similarities. The QR results of previous studies show that the association between maternal education and child growth differs along the conditional HAZ distribution ${ }^{(2,17,26,29)}$. An improvement in maternal education was associated with a dramatic increase in child $\mathrm{HAZ}^{(2,17)}$. In Sri Lanka, Aturupane et al. ${ }^{(26)}$ observed that the beneficial effect of maternal schooling on child height accrues disproportionately to children in the upper tail of the conditional nutritional distribution. Thus, maternal education has a beneficial effect on child nutritional status; however, the effect may be context specific.

Similarly, in the current analysis, maternal work status is positively related to the two lower quantiles in Nigeria and inversely related to all quantiles except the 25th in Kenya, with the effect being greatest at the lowest and uppermost parts of the conditional HAZ distribution. This implies that Kenyan mothers who are engaged in any form of work tend to have children who have lower HAZ relative to mothers who are not working. This negative effect may boil down to inadequate childcare due to limited time available to working mothers. Indeed, although it is a welldocumented fact that women who are working tend to have access to disposal income and therefore are able to provide nutritious food as well as other care services to their children ${ }^{(19,27-29)}$, the negative effect of maternal work on child health has also been observed previously. For example, studies have revealed that the most malnourished children have mothers who work outside their home ${ }^{(30,31)}$. The present analysis did not investigate the categories of employment and their effect on child growth; we are therefore unable to tell the independent effect of the various occupational groups on child HAZ. This is a limitation worth noting, as different occupations may have different effects.

Another important determinant of child linear growth is maternal nutrition, measured by BMI and height. In the current analysis, BMI tends to have significant positive effects throughout the conditional HAZ distribution in four out of the five countries. The largest effects occur in the upper quantiles, suggesting a disproportionate beneficial effect of BMI accruing to children in the upper tail of the conditional HAZ distribution. Thus, improving maternal nutrition may have a positive effect on children's linear growth, especially those in the upper end of HAZ distribution. Hence, interventions aimed at increasing maternal BMI qualitatively are likely to be more effective in improving the linear growth of children in the upper tail of HAZ distribution. Similarly, maternal height has increasing positive effects throughout the conditional HAZ distribution, with the largest effect occurring at the 50th quantile in all five countries. This suggests that improving maternal height may have a significant impact on child linear growth irrespective of the country of residence. These findings are consistent with previous studies. In India, BMI among other variables was found to have significantly larger effect on child linear growth ${ }^{(17)}$. A related study showed the differential effects of maternal nutrition at different points of the conditional distribution of the child anthropometric $Z$-scores ${ }^{(2)}$. Maternal height has also been found by previous studies to associate significantly and positively with child growth $^{(15,21)}$. The preceding discussion suggests the crucial role maternal nutrition plays in improving child 
nutritional status, even though the pathways through which this happens may be complex.

Furthermore, it is important to recognize that the differential effects of maternal characteristics on child linear growth as outlined above could be due to the notion that child growth and development are dependent on many factors, with some affecting some children more than others ${ }^{(47)}$. The impact can be positive as well as negative. Relating this to the present study, the findings give a sense that the response of the growth trajectories to maternal-level factors vary from one group of children to another. For example, in our analysis, maternal education has the greatest effect on the HAZ of children who are at higher risk (at lower end of the conditional HAZ distribution) of undernutrition relative to those who are at lesser risk. This may suggest that maternal education is more sensitive for children who have lower growth scores relative to children who have higher growth scores. Growth differences in response to maternal characteristics have been documented in the literature. A study that disentangled the growth parameters of size, velocity and tempo found that prenatal maternal characteristics were associated with different aspects of infant growth ${ }^{(48)}$. Although that study offered insights into the mechanisms governing infant growth, the pathways through which this effect occurs may not be straightforward, as it may be difficult to appreciate how education, for example, influences child growth directly without mediating factors.

\section{Strengths and limitations}

The present study is associated with a number of strengths. An important strength is the use of large nationally representative samples, thereby providing more robust estimates of observed associations. Additionally, the HAZ used in analyses as an outcome variable was objectively measured, reducing possible misclassification. The use of $\mathrm{QR}$ in the analysis is also an important methodological strength, as previous research mostly relies on OLS and can be misleading from a policy perspective. QR estimates enable us to get a comprehensive picture of the effects of DD and maternal characteristics on HAZ, which OLS is insufficient to illuminate. This is because the OLS techniques summarize the average relationship between a set of regressors and the outcome variable based on the conditional mean function ${ }^{(44,49)}$. This provides only a partial view of the relationship, as we might be interested in describing the relationship at different points in the conditional distribution of the outcome variable. The QR method is able to do this by providing a more detailed insight beyond the measures of central tendency (mean) and detecting sources of considerable heterogeneity in the effects of explanatory variable along the entire distribution of the outcome variable ${ }^{(44,50)}$.

There are also some limitations. One such limitation has to do with the cross-sectional nature of the data, which makes it difficult to disentangle potential reciprocal causations. The conclusions herein are therefore merely interpreted as associations between the predictor variables and the outcome variable. Another limitation is related to the child DD score used in the analysis. The key weakness of this method is that the mothers may not be able to report their children's food consumption accurately due to cognitive challenges such as lack of knowledge, forgetfulness and the interview situation ${ }^{(51)}$. There is evidence that the $24 \mathrm{~h}$ recall tends to underestimate food intake by about $10 \%$ relative to observed intake ${ }^{(52)}$; however, these cognitive challenges can be overcome by the use of probes by the interviewer, which has been well established as an effective means to recover foods that the respondent has not reported ${ }^{(51)}$. The DHS, like any other survey, has made probes an integral part of its interviewing protocols. Therefore, the DHS data collectors might have addressed any cognitive challenges of respondents during the data collection process.

\section{Conclusions and policy implications}

The current study uses QR to estimate the effects of child DD and maternal characteristics on child HAZ. We find that OLS estimates of DD and maternal-level factors on child HAZ, which effectively estimate the effects of intervention variables at the mean, can be misleading. For instance, the OLS regression did not indicate the presence of differential effects of DD on child HAZ; however, the QR models show that the largest effect of DD on HAZ occur at the lowest (5th) and the highest (90th) quantiles in Nigeria, while in DRC the effect is observed only at the 5th and 10th quantiles. Policy interventions to improve child nutrition need to be sensitive to this reality. Likewise, the OLS estimates of maternal education are misleading. While such estimates would lead one to believe that increase in maternal years of education is associated with strong nutritional improvement, the QR results indicate this is generally true at the lower end of the conditional HAZ distribution. The implication of this is that policy interventions to improve maternal education are likely to be more effective in raising the nutritional level of those children at the greatest risk of malnutrition. We find that maternal nutrition has a significant positive effect on child HAZ in all countries. This implies that interventions to improve maternal nutrition would have a substantial effect in raising the nutritional level of children.

\section{Acknowledgements}

Acknowledgements: The authors thank the DHS Program, the Ghana Health Service and the Ghana Statistical Service for the data used in this study. Financial support: This research received no specific grant from any funding 
agency in the public, commercial or not-for-profit sectors. Conflict of interest: None declared. Authorship: D.A.A. conceived and designed the study, interpreted the results, wrote the first draft of the manuscript and contributed to revision of the manuscript. Z.T.D. and D.A.A. analysed the data. Z.T.D., E.W.K.-M., B.M. and A.C.E. contributed to study design, data interpretation and critical revision of the manuscript. All authors read and approved the final version, and take responsibility for any issue that may arise from the publication of this manuscript. Ethics of human subject participation: DHS were approved by the national ethics committees of the respective countries, each participant gave written informed consent and parents consented to their children being measured before data were collected. The DHS Program granted the authors permission to use the anonymous data and further ethical clearance was not sought.

\section{References}

1. UNICEF (2013) Improving Child Nutrition: The Achievable Imperative for Global Progress. New York: UNICEF.

2. Uttamacharya U, Arokiasamy P \& Verma R (2013) Determinants of child anthropometrics in India: a quantile regression analysis. http://iussp.org/en/event/17/ programme/paper/3785 (accessed July 2016).

3. Rice LA, Sacco L, Hyder A et al. (2000) Malnutrition as an underlying cause of childhood deaths associated with infectious diseases in developing countries. Bull World Health Organ 78, 1207-1221.

4. Black RE, Allen LH, Bhutta ZA et al. (2008) Maternal and child undernutrition: global and regional exposures and health consequences. Lancet 371, 243-260.

5. Black RE, Victora CG, Walker SP et al.; Maternal and Child Nutrition Study Group (2013) Maternal and child undernutrition and overweight in low-income and middle-income countries. Lancet 382, 427-451.

6. Food and Nutrition Technical Assistance, Working Goup on Infant and Young Child Feeding Indicators (2006) Developing and Validating Simple Indicators of Dietary Quality and Energy Intake of Infants and Young Children in Developing Countries: Summary of Findings from Analysis of 10 Data Sets. Washington, DC: Academy for Educational Development.

7. Moursi MM, Arimond M, Dewey KG et al. (2008) Dietary diversity is a good predictor of the micronutrient density of the diet of 6 to 23-months-old children in Madagascar. J Nutr 138, 2448-2453.

8. Kennedy GL, Pedro MR, Seghieri C et al. (2007) Dietary diversity score is a useful indicator of micronutrient intake in non-breast-feeding Filipino children. J Nutr 137, 472-477.

9. Ogunba BO (2010) Diet diversity in complementary feeding and nutritional status of children aged 0 to 24 months in Osun State, Nigeria: a comparison of the urban and rural communities. http://can.sagepub.com/content/2/6/ 330.refs.html (accessed June 2016).

10. Rah JH, Akhter N, Semba RD et al. (2010) Low dietary diversity is a predictor of child stunting in rural Bangladesh. Eur J Clin Nutr 64, 1393-1398.

11. Ekesa BN, Blomme G \& Garming H (2011) Dietary diversity and nutritional status of pre-school children from musadependent households in Gitega (Burundi) and Butembo
(Democratic Republic of Congo). Afr J Food Agric Nutr Dev 11, 4896-4911.

12. World Health Organization (2009) Indicators for Assessing Infant and Young Child Feeding Practices. Part II: Measurement. Geneva: WHO.

13. Amugsi DA, Mittelmark MB \& Lartey A (2014) Dietary diversity is a predictor of acute malnutrition in rural but not in urban settings: evidence from Ghana. Br J Med Med Res 4, 4310-4324.

14. Arimond M \& Ruel MT (2004) Dietary diversity is associated with child nutritional status: evidence from 11 demographic and health surveys. J Nutr 134, 2579-2585.

15. Felisbino-Mendes MS, Villamor E \& Velasquez-Melendez G (2014) Association of maternal and child nutritional status in Brazil: a population based cross-sectional study. PLoS One 9, e87486.

16. Rachmi CN, Agho KE, Li M et al. (2016) Stunting, underweight and overweight in children aged $2.0-4.9$ years in Indonesia: prevalence trends and associated risk factors. PLoS One 11, e0154756.

17. Fenske N, Burns J, Hothorn T et al. (2013) Understanding child stunting in India: a comprehensive analysis of socio-economic, nutritional and environmental determinants using additive quantile regression. PLoS One 8 , e78692.

18. Subramanian SV, Ackerson LK, Davey Smith G et al. (2009) Association of maternal height with child mortality, anthropometric failure, and anemia in India. JAMA 301, 1691-1701.

19. Negash C, Whiting SJ, Henry CJ et al. (2015) Association between maternal and child nutritional status in Hula, rural southern Ethiopia: across sectional study. PLoS One 10, e0142301.

20. Aheto JM, Keegan TJ, Taylor BM et al. (2015) Childhood malnutrition and its determinants among under-five children in Ghana. Paediatr Perinat Epidemiol 29, 552-561.

21. Ozaltin E, Hill K \& Subramanian SV (2010) Association of maternal stature with offspring mortality, underweight, and stunting in low- to middle-income countries. JAMA $\mathbf{3 0 3}$, 1507-1516.

22. Sonkaria L, Zafer A, Gaur KL et al. (2014) Maternal factors associated with nutritional status of $1-5$ years children residing in field practice area of rural health training centre Naila, Jaipur (Rajasthan) India. Natl J Community Med 5, 283-287.

23. Finlay JE, Özaltin E \& Canning D (2011) The association of maternal age with infant mortality, child anthropometric failure, diarrhoea and anaemia for first births: evidence from 55 low- and middle-income countries. BMJ Open 1, e000226.

24. Fall CH, Sachdev HS, Osmond C et al.; COHORTS Investigators (2015) Association between maternal age at childbirth and child and adult outcomes in the offspring: a prospective study in five low-income and middle-income countries (COHORTS collaboration). Lancet Glob Health 3, e366-e377.

25. Thuita FM, Mwadime RKN \& Wang'ombe JK (2005) Child nutritional status and maternal factors in an urban slum in Nairobi, Kenya. East Afr Med J 82, 209-215.

26. Aturupane H, Deolalikar AB \& Gunewardena D (2008) The Determinants of Child Weight and Height in Sri Lanka A Quantile Regression Approach. Research Paper no. 2008/53. https://www.gov.uk/dfid-research-outputs/the-determinants-ofchild-weight-and-height-in-sri-lanka-a-quantile-regressionapproach (accessed June 2016).

27. Keino S, Plasqui G, Ettyang G et al. (2014) Determinants of stunting and overweight among young children and adolescents in sub-Saharan Africa. Food Nutr Bull 35, $167-178$. 
28. Abuya BA, Ciera J \& Kimani-Murage E (2012) Effect of mother's education on child's nutritional status in the slums of Nairobi. BMC Pediatr 12, 80.

29. Sharaf MF \& Rashadb AS (2016) Economic and SocioDemographic Determinants of Child Nutritional Status in Egypt: A Comprehensive Analysis using Quantile Regression. Approach. Working Paper no. 2016-04. http://www.ualberta. ca/ econwps/2016/wp2016-04.pdf (accessed June 2016).

30. Abbi R, Christian P, Guiral S et al. (1991) The impact of maternal work on the nutrition and health status of children. Food Nutr Bull 13, 20-24.

31. Popkin BM (1980) Time allocation of the mother and child nutrition. Ecol Food Nutr 9, 1-14

32. Lamontagne JF, Engle PL \& Zeitlin MF (1998) Maternal employment, child care, and nutritional status of 12-18-month-old children in Managua, Nicaragua. Soc Sci Med 46, 403-414.

33. Lü B, Zhai F, Jin S et al. (1999) Impact of maternal occupation on the dietary and nutritional status of preschool children. A case study in 8 provinces of China. Wei Sheng Yan Jiu 28, 47-49.

34. The DHS Program (n.d.) Demographic and Health Surveys. http://dhsprogram.com/data/available-datasets.cfm (accessed March 2016).

35. Ghana Statistical Service, Ghana Health Service \& ICF Macro (2009) Ghana Demographic and Health Survey 2008. Accra and Calverton, MD: GSS, GHS and ICF Macro.

36. Ministry of Health and Social Services \& Macro International Inc (2008) Namibia Demographic and Health Survey 2006-07. Windhoek and Calverton, MD: MoHSS and Macro International Inc.

37. Ghana Statistical Service, Ghana Health Service \& ICF International (2015) Ghana Demographic and Health Survey 2014. Rockville, MD: GSS, GHS and ICF International.

38. National Population Commission \& ICF International (2014) Nigeria Demographic and Health Survey 2013. Abuja and Rockville, MD: NPC and ICF International.

39. National Bureau of Statistics-Kenya \& ICF International (2015) 2014 KDHS Key Findings. Rockville, Maryland: KNBS and ICF International.

40. WHO Multicentre Growth Reference Study Group (2006) WHO Child Growth Standards based on length/height, weight and age. Acta Paediatr Suppl 450, 76-85.

41. World Health Organization (2001) Exclusive breastfeeding. http://www.who.int/nutrition/topics/exclusive_breastfeeding/ en/ (accessed June 2016).
42. US Agency for International Development (2012) Maternal Dietary Diversity and the Implications for Children's Diets in the Context of Food Security. Washington, DC: USAID.

43. World Health Organization (2010) Indicators for Assessing Infant and Young Child Feeding Practices. Part 3: Country Profiles. Geneva: WHO; available at http://www.unicef.org/ nutrition/files/IYCF_Indicators_part_III_country_profiles.pdf

44. Koenker R \& Bassett G (1978) Regression quantiles. Econometrica 46, 33-50.

45. Amugsi DA, Dimbuene ZT, Bakibinga P et al. (2016) Dietary diversity, socioeconomic status and maternal body mass index (BMI): quantile regression analysis of nationally representative data from Ghana, Namibia and Sao Tome and Principe. BMJ Open 6, e012615.

46. Ntab B, Simondon KB, Milet J et al. (2005) young child feeding index is not associated with either height-for-age or height velocity in rural Senegalese children. $J$ Nutr $\mathbf{1 3 5}$, 457-464.

47. Business and Technology Education Council (n.d.) BTEC First Children's Care, Learning and Development: Understanding children's development. https://www.pearsonschoolsandfe colleges.co.uk/FEAndVocational/Childcare/BTEC/BTECFirst ChildrensCareLearningandDevelop/Samples/SampleMaterial/ UCD\%20Unit\%201.pdf (accessed November 2016).

48. Pizzi C, Cole TJ, Richiardi L et al. (2014) Prenatal influences on size, velocity and tempo of infant growth: findings from three contemporary cohorts. PLoS One 9, e90291.

49. Lê Cook B \& Manning WG (2013) Thinking beyond the mean: a practical guide for using quantile regression methods for health services research. Shanghai Arch Psychiatry 25, 55-59.

50. Harry H, Friedrich L \& Mark S (2014) Quantile regression analysis and other alternatives to ordinary least squares regression: a methodological comparison on corporal punishment. Eur J Res Meth Behav Soc Sci 10, 81-91.

51. Thompson FE \& Subar AF (2013) Dietary assessment methodology. In Nutrition in the Prevention and Treatment of Disease, 3rd ed., pp. 6-46 [AM Coulston, CJ Boushey and MG Ferruzzi, editors]. London: Elsevier Inc.

52. Australasian Child and Adolescent Obesity Research Network (2006) Dietary intake assessment - 24-hr recall. http:// www.acaorn.org.au/streams/nutrition/assessment-methods/ 24hr-recall.php (accessed March 2016). 